1

2

3

\title{
Toward the development of a hydrofoil tailored to passively reduce its lift response to fluid load
}

\author{
L. Marimon Giovannettia , J. Banks ${ }^{\mathrm{a}}$, M. Ledri, S. R. Turnock ${ }^{\mathrm{a}}$, S. W. Boyd ${ }^{\mathrm{a}}$ \\ ${ }^{a}$ Faculty of Engineering and the Environment, Fluid Structure Interaction Group, \\ University of Southampton, UK
}

\begin{abstract}
The objective of this research is to explore the possibility of using Passive Adaptive Composite (PAC) on structures to help control the lift generated by hydrofoils on boats such as the International Moth. Intorducing composite fibres oriented at off-principal axis angles, allow a foil to passively control its pitch angle to reduce the lift generated at higher boat speeds helping to achieve a stable flight in a wide range of weather conditions. PAC utilises the inherent flexibility of a composite structure to induce a twist response under bending load which could be used to minimise the use of active control systems, or even improve the dynamic response of foils in waves. However, to design flexible foils requires numerical and experimental tools to assess the complex fluid structure interactions involved. This paper eveluates a simplified hydrofoil geometry designed to reduce its lift coefficient with increased flow speed. A coupled Computational Fluid Dynamics (CFD) and Finite Element Analysis (FEA) methodology is presented to predict flexible foil performance. Validation of these numerical tools is achieved through the use of wind tunnel experiments including full field deformation measurements. Twist deformations resulted in a reduction in the effective angle of attack by approximately $30 \%$ at higher flow speeds reducing the foil lift and drag significantly.
\end{abstract}

Keywords: Fluid Structure Interaction, Passive Adaptive Composites, High Performance sailing

Email address: L.Marimon-Giovannetti@soton.ac.uk (L. Marimon Giovannetti) 


\begin{tabular}{|c|c|c|}
\hline \multicolumn{3}{|c|}{ Nomenclature } \\
\hline Symbol & Definition & [unit] \\
\hline 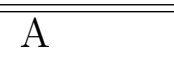 & Area & {$\left[\mathrm{m}^{2}\right]$} \\
\hline$A_{i j}$ & In-plane stiffness $[\mathbf{A}]$ & \\
\hline$B_{i j}$ & Bending-extension coupling $[\mathbf{B}]$ & \\
\hline $\mathrm{C}$ & Asymmetric aerofoil constant & \\
\hline CFD & Computational Fluid Dynamics & \\
\hline$C_{D}$ & Drag coefficient & \\
\hline$C_{L}$ & Lift coefficient & \\
\hline$D_{i j}$ & Bending stiffness $[\mathbf{D}]$ & \\
\hline DIC & Digital Image Correlation & \\
\hline DSBT & Dirrential Stiffness Bend-Twist & \\
\hline FEA & Finite Element Analysis & \\
\hline FSI & Fluid Structure Interaction & \\
\hline $\mathrm{K}$ & Lift curve slope & \\
\hline $\mathrm{k}$ & Number of layer & \\
\hline$k_{x, y, x y}$ & Laminate curvature & \\
\hline $\mathrm{L}$ & Lift force & {$[\mathrm{N}]$} \\
\hline$M_{i j}$ & Moments per unit length & {$[\mathrm{Nm}]$} \\
\hline$N_{i j}$ & Forces & {$[\mathrm{N}]$} \\
\hline $\mathrm{PAC}$ & Passive Adaptive Composites & \\
\hline PIV & Particle Image Velocimetry & \\
\hline$Q_{i j}$ & Stiffness matrix components & \\
\hline $\mathrm{t}$ & Layer thickness & {$[\mathrm{mm}]$} \\
\hline V & Wind speed & {$\left[\mathrm{ms}^{-1}\right]$} \\
\hline W & Weight & {$[\mathrm{kg}]$} \\
\hline$y^{+}$ & y-plus value & \\
\hline $\mathrm{z}$ & Layer distance from the ref. plane & {$[\mathrm{mm}]$} \\
\hline$\alpha$ & Angle of attack & [deg] \\
\hline$\gamma_{x y}$ & Shear strain & \\
\hline$\Delta t$ & Exposure time PIV camera & {$[\mu s]$} \\
\hline$\frac{\partial \alpha}{\partial I}$ & Bend-twist coupling term & \\
\hline$\epsilon$ & Strain & \\
\hline
\end{tabular}




$\begin{array}{lll}\rho & \text { Density } & {\left[\mathrm{kgm}^{-3}\right]} \\ \sigma & \text { Stress } & {[\mathrm{MPa}]} \\ \tau_{x y} & \text { Shear stress } & {[\mathrm{MPa}]}\end{array}$

\section{Introduction}

The International Moth is a single-handed ultra-lightweight foiling development class boat (International Moth Class, 2013). When foiling the mass of the boat and helm remains constant but the lift produced by the daggerboard and rudder T-foils increases with the square of the boat speed. The daggerboard T-foil is formed of two elements with an adjustable rear flap to modify the lift produced based on the vessel ride height. This constant movement of the rear foil changes the viscous drag of the whole foil section. Meanwhile, the angle of attack of the forward main element is slightly adjusted with dynamic body movements of the helm. The importance of being a lightweight sailing boat enhanced the use of composite materials. Using composite materials it is possible to design a structure tailored to a certain load, in this case the mass of the boat plus the crew. Introducing plies oriented at angles different than zero, 90 or 45 degrees that are normally used in quasi-isotropic structures, it is possible to change the response of a composite structure under load.

Moreover, with the recent increase of foiling boats there is still a lack of accurate measures of structural response and shape of the hydrofoils. This gives rise to scientific questions on whether there is a manufacturing consistency between the port and starboard foil on a catamaran or different batches of foils on mono-hulls.

The aim of the current research is therefore to develop numerical and experimental tools capable of accurately describing the structural response of a foil under fluid-load and to design and develop a foil structure tailored to decrease its lift coefficient as the flow speed increases. In order to do so a coupled CFD and FEA methodology is developed and validated using full-field measurement techniques within a wind tunnel environment.

Those techniques are described in section 2 together with the advantages of developing a robust and repeatible Fluid Structure Interaction (FSI) experimental methodology. The FSI experimental technique was initially developed at the University of Southampton (Banks et al., 2015; Marimon Giovannetti et al., 2017; Marimon Giovannetti, 2017) and provides not only 
a measure of synchronised structural deformation and fluid response but also the uncertainty values associated with coupling the two optical systems.

Moreover, in section 3 two techniques that can be used to change the performance profile of an hydrofoil are described, namely Passive Adaptive Composites (PAC) that tailors the response of a structure by changing the orientation of the composite plies (Veers et al., 1998) and Differential Stiffness Bend-Twist (DSBT) that utileses the internal stiffness of a structure to change the aero-hydrodynamic response to fluid load (Raither et al., 2012). These two techniques, if used in parallel, can substantially change the effective angle of attack of a foil structure under load. The current research presents the first steps in investigating the possibilities of applying those techniques to the design of a daggerboard T-foil of the International Moth. PAC have been researched in applications for wind-turbine blades (De Goeij et al., 1999; Lin and Lai, 2010; Maheri and Isikveren, 2009), tidal turbines (Nicholls-Lee et al., 2009; Barber, 2017), propeller blades (Khan et al., 2000; Young and Motley, 2011) and Micro Air Vehicles (Hu et al., 2008; Tamai et al., 2008), so it is now possible to use the knowledge from other applications for high performance sailing boats.

After the two background sections, an idealised section that can be adapted in future research to high speeds boats such as the International Moth is presented. Utilising the inherent flexibility of PAC at high speeds and relatively large tip deflections the angle of attack can be passively reduced to decrease the induced drag. Twisting an aerofoil section toward feather indeed reduces the effective angle of attack of the foil. The equations of an analytical model that relates the lift force to the plies orientation within the structure are also described.

The design of the flexible aerofoil is presented in section 5. The position and layup of the internal stiffener are presented as well as the manufacturing techniques.

Finally, the full-scale experimental and numerical setup as well as the results from an idealised section are presented to demonstrate the passiveadaptive response to fluid load. This research merely represents the findings on FSI of a full-scale flexible model. Those results can be used in future projects as base to build a main element foil of the International Moth. 


\section{Background on FSI experimental measures}

Within the available literature there is a lack of analytical solutions and experimental measures of FSI problems (de Borst et al., 2013; Hou et al., 2012). Therefore, research in this area has mainly focused on coupled numerical solutions or approximations extensively utilising Blade Element Momentum (BEM) theory, Computational Fluid Dynamics (CFD) and structural Finite Element Analysis (FEA) simulations. Even though numerical studies have been extensive, especially in recent years with the increase in computer power, there is a lack of experimental validation cases for FSI problems and, for the limited cases that there are, the uncertainty in measurements is often unknown.

The first FSI experiments were developed with known experimental techniques re-adapted to allow the capture of both structural deformations and load capture. Fedorov (2012) presents a numerical and experimental approach where the effects of loads on a composite bend-twist full-scale wind turbine blade are measured. In particular they measured deflection and twist using Digital Image Correlation (DIC). However, his research lacks the dynamic coupling from the aerodynamic forces, as a known hydraulic load was applied whereas in a complete FSI experiment the aerodynamic force will actually change due to fluid-induced deformation and twist.

High speed cameras and laser Doppler vibrometers were employed to measure the pitch motion of a flexible hydrofoil and the areas of cavitation by Ducoin et al. (2012). These experiments present the displacement and pitch angle for the tip section of a two-dimensional hydrofoil under real flow conditions. The study developed by Ducoin et al. (2012) provides useful validation material for cavitation simulations, including structural deformation, but does not provide the hydrodynamic forces or flow field information to assess non-cavitating CFD models. Malijaarsl and Kaminski (2015) present a review of the published studies on flexible propellers. The possibility of using composite propellers to reduce cavitation problems is addressed, but they identify a need for experiments to validate the hydro-elastic numerical simulations, as well as measurements of the deformed shape of flexible propellers. More recently, Barber (2017) tested in a flume-tank two sets of PAC marine hydrokinetic (MHK) turbine blades alongside one non-adaptive composite and a set of aluminium blade. The magnitude and direction of the torque on the rotor applied by the flow were measured with two load cells. The flow velocity was recorded so that the capacity for power generation 
could be calculated. Those tests were however performed at model scale, meaning that the bend-twist coupling of the composite structure could not be related to a full-scale MHK turbine as the composite blades were composed of a flat carbon fibre spar, twisted to follow the chord line of the blade geometry and a semi-flexible urethane body cast around the spar to create the hydrodynamic blade shape.

To assess the validity of numerical FSI simulations we require the ability to measure the influence of fluid load on the structural response. It is important that this is conducted in a controlled manner, to provide data with known uncertainties for comparison with numerical FSI simulations. CFD is often validated in isolation using flow field data captured with Particle Image Velocimetry (PIV) and measured aero-hydrodynamic forces (Jones et al., 2008). Similarly FEA models can be validated against full-field deformation measurements acquired using DIC (Siddiqui, 2014).

In order to fully capture the behaviour of a structure or a fluid under investigation without interfering with it, full-field non-contact measurement techniques can be used. These techniques have been employed extensively in the last two decades, as described by Raffel et al. (2007), Rastogi and Hack (2012), and Grediac and Hild (2012), and are capable of recording the data necessary to describe the surface's structural behaviour or the flow surrounding an object without modifying the nature of the structure or of the flow itself. These capabilities, which are beneficial with respect to point measurement techniques, together with the recent developments in sensor cameras and computer power, has permitted an increase in use of Digital Image Correlation and Particle Image Velocimetry in recent years.

Particle Image Velocimetry (PIV) and Digital Image Correlation (DIC) are both full field, non-contact, light based optical techniques and have been extensively used in a wide variety of experiments separately since the 1980s (Wieneke, 2014; Chen et al., 2013).

Digital Image Correlation (DIC) is a measurement technique used to analyse problems in solid mechanics. It is a white light full-field non-contact measurement technique (Tang et al., 2012) which involves the use of CCD digital cameras that register a series of images of a surface on which a randomised speckle pattern is applied. From these images, the displacement, the strain, and the stress of the specimen can be measured, using a correlation algorithm, tracking the motion of each speckle within the field of view (Rastogi and Hack, 2012). The speckle pattern is applied to the surface, providing a grey-level distribution which should be adequately illuminated in order to 
achieve a smooth histogram. This technique has been used for different scale problems, from high magnification (Crammond et al., 2013) to large scale structures (Mccormick, 2012). Within the DIC software, the speckle pattern is mapped to calculate its deformation, allowing the displacements and strains of the underlying structure to be measured. DIC has previously been used to measure foil deflections under fluid loads, with a technique developed at the University of Southampton (Banks et al., 2015; Marimon Giovannetti et al., 2017). The key advantages are the use of simple equipment (i.e. cameras, lenses, light and computer), the fact that is a non-contact measurement and its high fidelity of precision (Tang et al., 2012). Within the DIC software, the correlation between speckle pattern in subsequent images is used to determine how the structure has deformed in time, thereby allowing the derivation of the full-field deflections and strains of the investigated object in a dynamic environment (Rastogi and Hack, 2012). The use of two cameras, in a stereo configuration, allows for the measurement of deformations both in the plane normal to the camera and out-of-plane.

Measuring the deformation and twist of a foil under known static load allows the assessment of its bending and torsional stiffness (Banks et al., 2016). Once the structural properties of the foil are known, it is possible to understand the effects of bending and twisting of the foil on the overall sailing performances. For the International Moth, shown in Figure 1, the change in lift coefficient with angle of attack for a Vendor T-foil was presented by Beaver and Zseleczky (2009). 


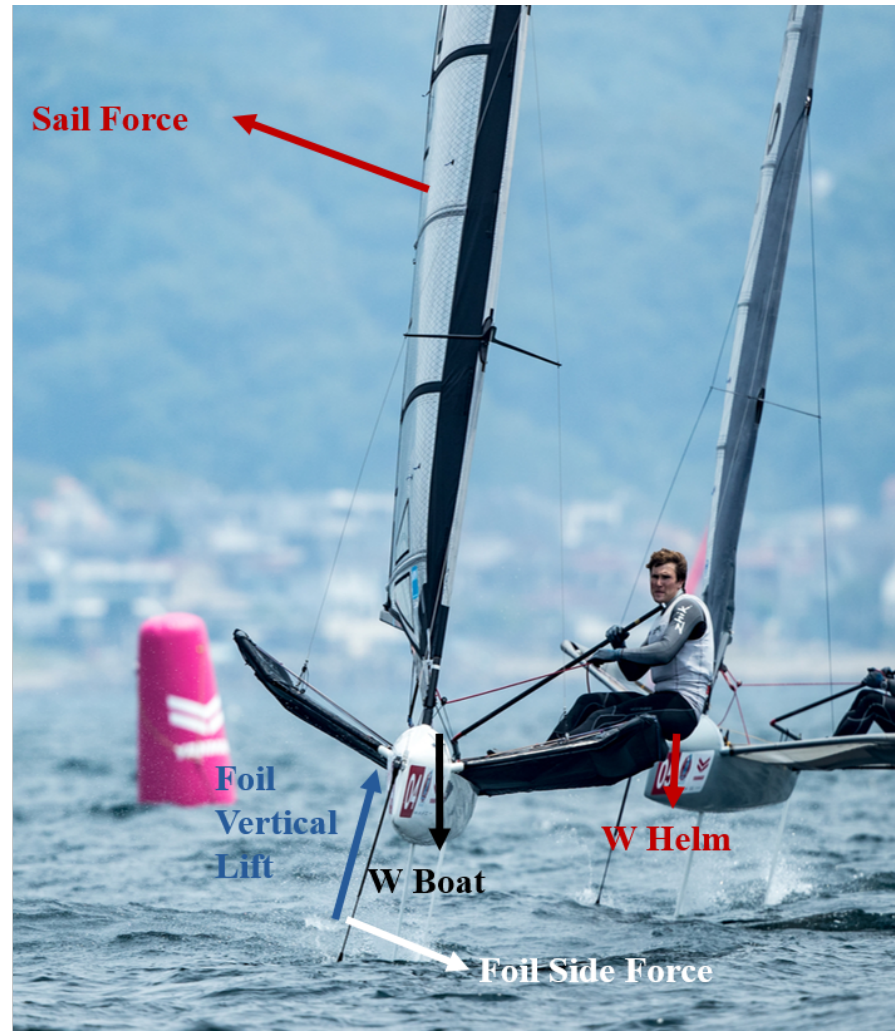

Figure 1: Forces acting on an International Moth whilst sailing.

Having investigated the tip deflection and twist in characteristic sailing loads during a laboratory test, as described by Banks et al. (2016), it was possible to assess that the tip deflection reduces the component of lift in the vertical direction as each end of the T-foil curves upward. Furthermore, the twist deformation associated with a downstream shift in centre of pressure (i.e. a $8 \%$ chord distance between the shear centre and the centre of pressure), reduces the local angle of attack by 0.23 degrees resulting in a $5 \%$ decrease in the generated lift force at take-off speed. It is clear that even under steady sailing loads the foil structural characteristics for a quasi-isotropic composite will have a noticeable effect on the performances of the boat.

\section{Background on bend-twist coupling}

In developing the design of a single-element foil that can twist toward feather with increased flow-speed, two bent-twist techniques are brought to- 
gether: the bend-twist coupling due to the orientation of the plies utilising Passive Adaptive Composites and the bend-twist coupling due to stiffness variation along the aerofoil chord utilising Differential Stiffness Bend-Twist (DSBT).

PAC presents fibres oriented in the same direction at opposite sides of the neutral axis, introducing an interaction between the hydrodynamic forces and the laminate curvatures and between the hydrodynamic moments and the laminate strains. Wind turbine blade design and adaptivity were initially investigated during the $70_{s}$ and $80_{s}$ as a way to alleviate tip loads during wind gusts. Using the anisotropy of the material, it is possible to design components presenting elastic couplings that will enhance the performance of the whole structure (Fedorov, 2012; Veers et al., 1998). In those structures, the relationship of the stresses and strains must take into consideration the complete stiffness matrix as the stresses $(\sigma$ and $\tau)$ and strains $(\epsilon$ and $\gamma)$ are coupled to $\sigma_{1}$ and/or $\sigma_{2}$ in the two principal directions, leading to:

$$
\left[\begin{array}{c}
\sigma_{x} \\
\sigma_{y} \\
\tau_{x y}
\end{array}\right]=\left[\begin{array}{lll}
\bar{Q}_{11} & \bar{Q}_{12} & \bar{Q}_{16} \\
\bar{Q}_{12} & \bar{Q}_{22} & \bar{Q}_{26} \\
\bar{Q}_{16} & \bar{Q}_{26} & \bar{Q}_{66}
\end{array}\right]\left[\begin{array}{c}
\epsilon_{x} \\
\epsilon_{y} \\
\gamma_{x y}
\end{array}\right]
$$

where $\mathbf{Q}_{\mathrm{ij}}$ represents the stiffness matrix in principal axis. Changing the ply angle in each lamina influences the stiffness matrix $\mathbf{Q}$ as the material axis is not aligned with the laminate axis. Under fluid loading, the forces and moments experienced by the composite will be related to the strains at laminate level as:

$$
\left[\begin{array}{c}
N_{x} \\
N_{y} \\
N_{x y} \\
M_{x} \\
M_{y} \\
M_{x y}
\end{array}\right]=\left[\begin{array}{cc}
\mathbf{A} & \mathbf{B} \\
\mathbf{B} & \mathbf{D}
\end{array}\right]\left[\begin{array}{c}
\epsilon_{x} \\
\epsilon_{y} \\
\gamma_{x y} \\
k_{x} \\
k_{y} \\
k_{x y}
\end{array}\right]
$$

where $N_{x}, N_{y}, N_{x y}$ are the fluid forces, $M_{x}, M_{y}, M_{x y}$ are the fluid moments, $k_{x}, k_{y}, k_{x y}$ are the laminate curvatures and

$$
A_{i j}=\sum_{k=1}^{N}\left(\bar{Q}_{i j}\right)_{k} t_{k} ; i, j=1,2,6
$$

represents the in-plane stiffness of the laminate (and presents values also for 
symmetric and balanced layups)

$$
B_{i j}=\sum_{k=1}^{N}\left(\bar{Q}_{i j}\right)_{k} t_{k} \bar{z}_{k} ; i, j=1,2,6
$$

represents the bending and/or extension coupling (i.e. the coupling term between moments and direct strains and forces and curvatures) and

$$
D_{i j}=\sum_{k=1}^{N}\left(\bar{Q}_{i j}\right)_{k}\left(t_{k} \bar{z}_{k}^{2}+\frac{t_{k}^{3}}{12}\right) ; i, j=1,2,6
$$

represents the bending stiffness of the component. In equations (3-5) $k$ is the layer number and $t_{k}$ is the thickness of the $k^{t h}$ layer. In equations (45) $\bar{z}_{k}$ is the distance from the mid-plane to the centroid of the $k^{t h}$ layer. In order to correctly design Passive Adaptive Composites, it is necessary to account for the $\mathbf{B}_{\mathbf{i j}}$ matrix as it gives the coupling terms for the bending-twist and extension-twist from the fluid-loads to the laminate structure. The $\mathbf{B}_{\mathbf{i j}}$ matrix for symmetric and balanced laminate structures is zero as there are no coupling terms between moments and direct strains. The bend-twist coupling is deriving from plies oriented at the same angle at each side of the neutral axis. The oriented plies allow to achieve tailored designs for an expected load, inducing a twist on the hydrofoil section (Marimon Giovannetti, 2017).

DSBT has been introduced initially in (Raither et al., 2012) with a conceptual investigation of a beam-like structure and its response to flexural load with different web-stiffness. By stiffening one side of the web more than the other, it is possible to induce twist to the structure. This concept is particularly important in hydrofoil structures and their hydro-elastic behaviour. Indeed, by stiffening the leading edge of a foil, the shear centre is moved forward, away from the centre of pressure, resulting in a structure twisting toward feather under bending load.

Changing the stiffness along the aerofoil chord to modify the shear centre position with respect to the centre of pressure is further analysed in (Herath et al., 2015) as a way of load alleviation in wind turbine blades.

Investigating the literature available to date on both techniques is important, during the development of the new foil-design, to understand the influence of both stiffness and passive adaptivity on the changes in effective angle of attack. 


\section{Optimised design for bend-twist coupling}

Conventional foil design assume a rigid shape, therefore the lift and drag coefficients are measured in experimental and numerical simulations for rigid sections. However, once analysing composite flexible foils, it is important to understand the effects of deflections on performance outcome. The International Moth horizontal foil provides the lift necessary to counteract the crew-hull weight, as presented in Figure 1. The produced force is dependent on the pitch angle as well as the boat speed squared. It is formed of a fixed and a moving elevator, as shown in Figure 2. In current foil design, both the fixed foil and elevator are made of quasi-isotropic composite material and the lift coefficient is controlled by constantly changing the flap angle, and therefore section shape, with the bow-wand (Figure 3) to maintain a constant lift force for different boat speeds. The sensor wand is passively pushed by the water as the ride height changes and the elevator is moved accordingly via a road in the vertical element on the foil to maintain a sufficient level of lift to sustain the crew and boat weight.

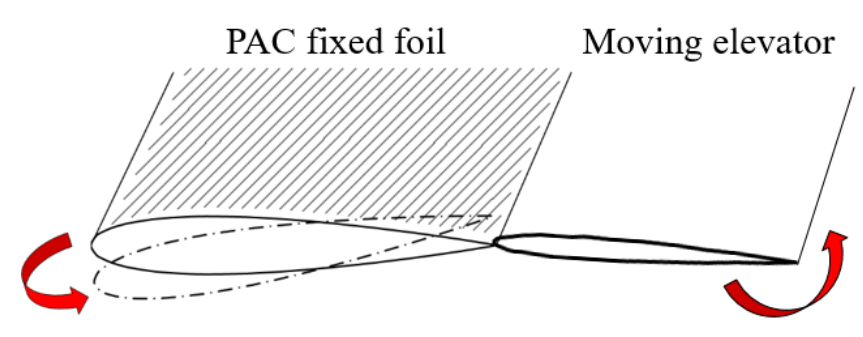

Figure 2: Idealised Passive Adaptive Composite main foil associated with moving elevator. In increased boat speeds the main element would twist toward feather and the elevator would reduce the total camber of the foil, following the red arrows. At current stage both the main element and the flap are built as quasi-isotropic structures. 


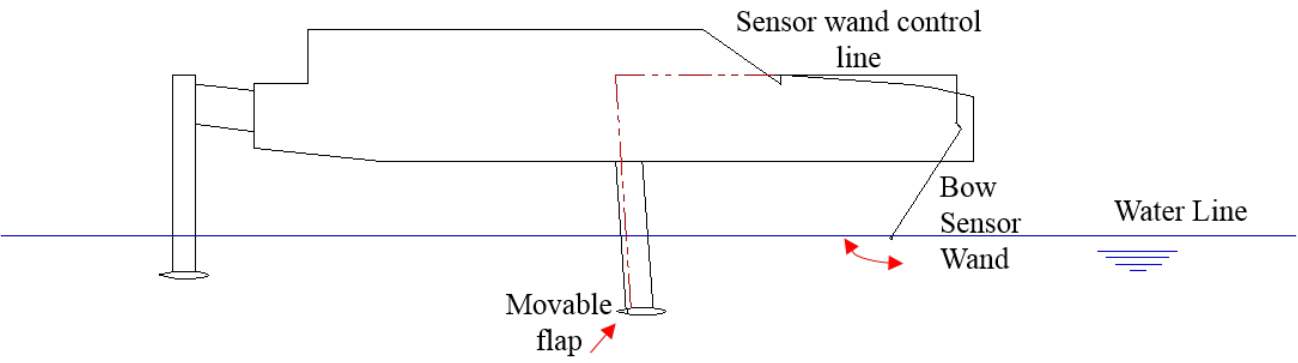

Figure 3: Bow wand sensor schematics showing the control line that moves the flap via a pushing rod.

An ideal lift profile aims to achieve a constant level of lift, resisting the constant mass of the sailor and the boat, at a chosen take-off speed, Figure 4. In current International moth design this is achieved by constantly adjusting the rear flap and therefore the camber of the overall section. The present research aims to investigate the possibility of passively changing the angle of attack of the main foil utilising bend-twist coupling techniques of composite materials, thus reducing the number of movements needed by the rear flap.



Figure 4: Ideal lift over velocity profile for a hydrofoil section achieved in the International moth by constantly changing the chamber of the foil with the movable rear flap.

In order to achieve a constant lift-curve, not only should the angle of 
attack change substantially but also the shape of the aerofoil, so as to decrease $C_{L}$ linearly over $V^{2}$. The blue arrow in Figure 5 represents a way in which the hydrofoil could achieve a constant lift value, combining a PAC main foil with a rear elevator to change the shape of the overall foil at different boat speeds.



Figure 5: Schematic of potential ways to reduce lift coefficient. Ideal lift coefficient curves for cambered and symmetric sections. The arrows show the influence of using PAC composites that change the angle of attack, the change in section shape that can be achieved with multi-elements foils or flaps, effectively reducing the camber, and a combination of the PAC and multi-element foils.

Figure 5 shows two typical lift coefficient curves for a cambered and a symmetric section. Using passive adaptivity it is possible to change the angle of attack, decreasing the $C_{L}$ value with increasing speed. This leads to a linear variation of lift with velocity, however, passive adaptive coupling requires an increase in tip deflection, and therefore lift force, to achieve a change in angle of attack. Therefore, bend-twist coupling is not able to achieve a constant lift force alone and the change in shape given by the rear 
flap is still needed. Decreasing the lift coefficient with speed at high angles of attack allows the delay of the stall angle, twisting the aerofoil toward feather. The ability to reduce the lift coefficient with increased flow speed allows to reduce also the induced drag, as the induced drag decreases proportionally to a decrease in angle of attack. Presenting a PAC main foil leads to less movements of the flap element, therefore leading to a reduction of viscous resistance, associated with the change in shape. For a hydrofoil design the effectiveness of passive adaptivity at low angles of attack (pitch angles) is important, as the rake has a limited range of angles adjustments.

The PAC change in angle of attack in Figure 5 can be described in an analytical approach analysing the lift equation

$$
L=\frac{1}{2} \rho A V^{2} C_{L}
$$

and the proportionality between the lift coefficient $C_{L}$ and the angle of attack $\alpha$, where $\alpha$ is formed of $\left(\alpha_{0} \pm \frac{\partial \alpha}{\partial L} L\right)$. In this case $\alpha_{0}$ is the set angle of attack and $\frac{\partial \alpha}{\partial L}$ is the level of twist achievable with the bend-twist coupling. The positive or negative sign of $\frac{\partial \alpha}{\partial L}$ depends on the orientation of the passiveadaptive plies, i.e. if it changes the angle of attack toward feather or toward stall. Therefore, rearranging the lift Equation (6) it is possible to obtain the lift force as a function of the bend-twist coupling value. For:

$$
C_{L}=K\left(\alpha_{0} \pm \frac{\partial \alpha}{\partial L} L\right)+C
$$

the lift force can be calculated as:

$$
L=\frac{\frac{1}{2} \rho A V^{2} K \alpha_{0}+C}{1 \pm \frac{1}{2} \rho A V^{2} K \frac{\partial \alpha}{\partial L}}
$$

where $\rho$ is the fluid density, $A$ is the aerofoil surface area, $V$ is the flow speed, $K$ is a constant defined from the shape of the aerofoil and is derived from the lift coefficient over angle of attack ratio, and $C$ is the camber constant for asymmetric aerofoils.

\section{Design of a PAC foil}

From herein an idealised flexible aerofoil is described and analysed numerically and experimentally. The purpose of this study is to understand the 
effects of introducing a PAC stiffener inside an aero-hydrodynamic structure capable of changing the performances of said structures with the introduction of bend-twist coupling plies.

In order to achieve a level of twist high enough to reduce the rate of change of lift with speed, it is necessary to maximise the distance between the shear centre and the centre of pressure, and that the PAC oriented plies should lie as far apart as possible from the neutral axis. When the bending and torsional stiffness are increased, a reduction in coupling effects is seen. This leads to the necessity of choosing a C-beam internal stiffener which enables to use the benefits of both bend-twist coupling techniques described in section 3.

A NACA 2412 is chosen as the base of the flexible aerofoil due to its similarity to daggerboard sections and for being easy to reproduce. Investigating a range of foiling boats, an ideal pitch angle of $3^{\circ}$ is often set at take-off speed as a trade-off between lift and drag. The flexible aerofoil was designed with a NACA 2412 set at $3^{\circ}$ at the tip. As the amount of twist increases from the root to the tip, a section shape with no-camber is chosen at the root (i.e. NACA0015) to create a small amount of lift in the root area. At half-span a NACA1412 section is introduced with a pre-twist angle of 1.5 degrees. This section shape is introduced to allow a smooth transition between a NACA0015 and a NACA2412. The section shapes and the internal C-beam can be seen in Figure 6.

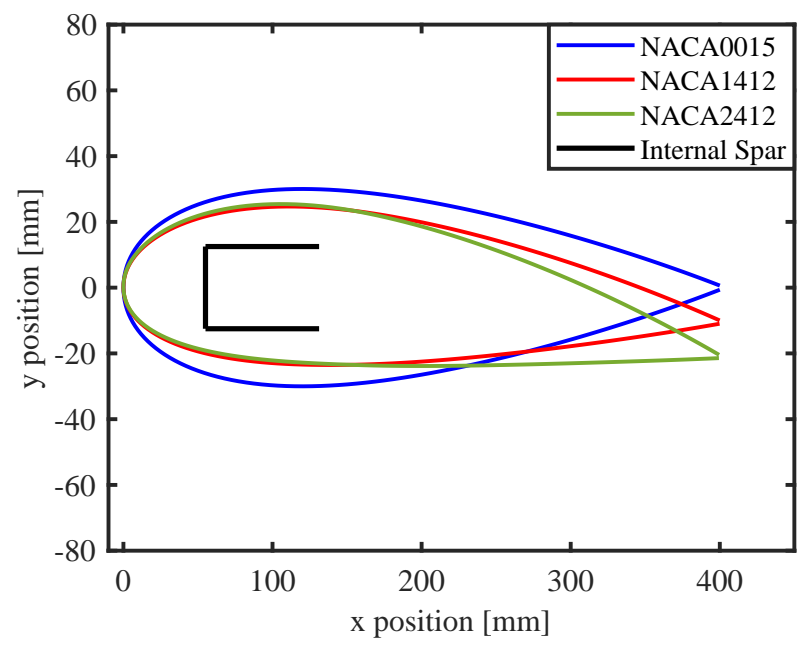

Figure 6: Section shapes of the flexible aerofoil under investigation. 
The aerofoil under investigation therefore is a combination of NACA 0015, NACA 1412 and NACA 2412 sections containing a load-carrying beam, an aerofoil-shaped foam-rib structure and a layer of Mylar to transfer the aerodynamic loading to the foam and the beam, see Figure 7. The manufacturing process of C-beam structure involved the use of unidirectional (UD) prepreg carbon and E-glass fibres (SE 84LV (Gurit, 2012; PRF Composite Materials, 2015)). The aerodynamic aerofoil foam structure manufacturing process was carried out using the hotwire technique to cut the foam to the desired shape. The leading and trailing edge foam structures were cut as a linear interpolation between the NACA 0015 and the NACA 2412 section shapes. All the foam parts were joined together by means of a two-component epoxy adhesive and the Mylar was then added to the structure.
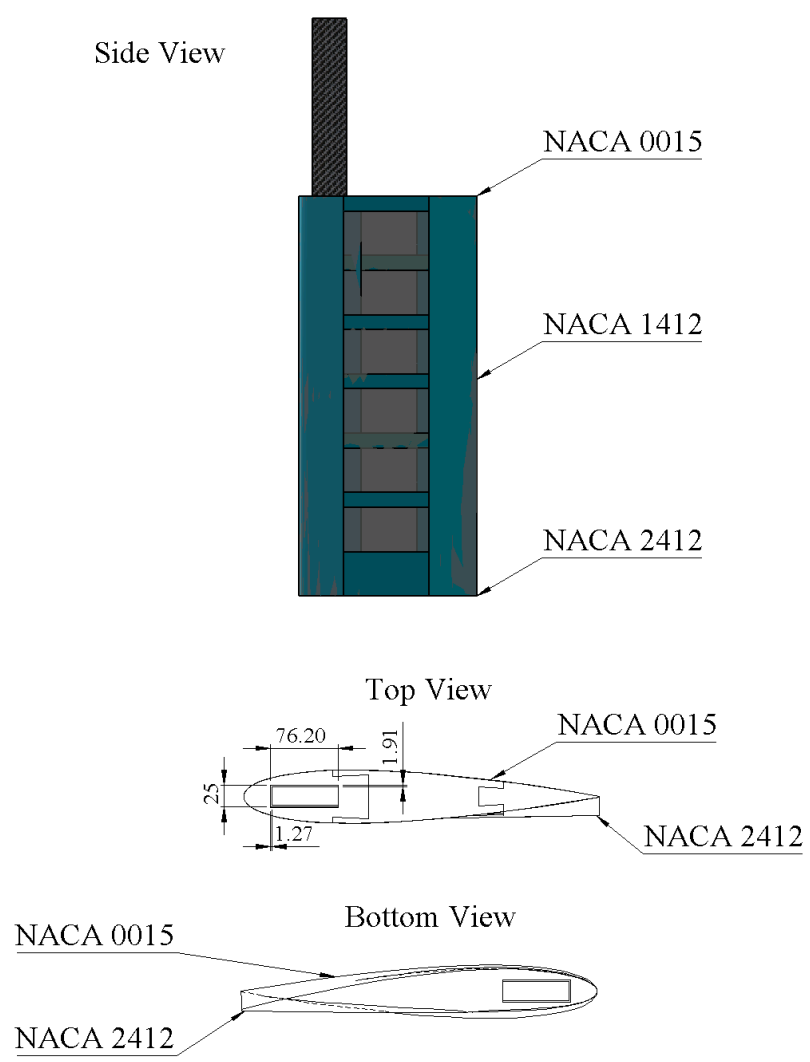

Figure 7: Foil technical drawing showing the different section shapes and the principal dimensions. All the dimensions shown are in millimetres. 


\section{Numerical and experimental set-up}

Given the high costs involved in experimental campaigns, it was necessary to achieve enough confidence in the response of the foil prior to entering the wind tunnel. Therefore, the numerical tools, validated against experimental values for an earlier design case, were used as design and developing tools to ensure a tailored response to load. The aerofoil shown in Figure 7 was modelled in the numerical environment. The structural model was solved using the numerical software ABAQUS 6.14, coupled to the fluid solver Star-CCM+ 11.0.2 through the Co-Simulation Engine (CSE). The co-simulation between ABAQUS and Star-CCM+ permits the creation of an interaction between the two domains through a common physical interface surface - the aerofoil surface. The interface conditions are the compatibility of the kinematics and tractions at the fluid-structure interface. The structure domain is in motion and its motion follows the material properties of the PAC beam. On the interface surface the data are mapped from the fluid-solver to the structural solver and vice-versa. Modelling the structural part in motion is known as the Lagrangian description of the structural motion. As the structure moves through space, the shape of the fluid sub-domain changes to conform to the motion of the structure. The motion of the fluid mechanics domain needs to be accounted for in the differential equations and boundary conditions. One of the most well-known methods used to capture the interaction between the structures and fluid is the Arbitrary Lagrangian Eulerian method (ALE) developed by Donea et al. (1982). ALE follows the equation of motion in a moving frame. This allows a control of the mesh geometry independently from the material geometry (Sigrist, 2015). In Star-ccm+ an automatic moving mesh scheme is generated to move the model points where the motions of the internal nodes is determined by solving the structural equations of elasticity (Star-ccm+, 2015). In the time-domain, the approach chosen to model the FSI coupling between the structure and the aerodynamic loading for the wind-tunnel model is a dynamic implicit solution, as there is a strong physical coupling deriving from the highly flexible aerofoil. In the implicit iterative approach, the fields are exchanged multiple times per coupling step until an overall equilibrium is achieved prior to advancing to the next coupling step. In the co-simulation the Mylar sheet surface, as well as the tip of the aerofoil and $50 \mathrm{~mm}$ of the PAC beam exposed to the wind, are chosen as interface regions, as can be seen in Figure 8. The same surface must be present and defined with the same name in the two solvers. The nodal posi- 
tion and the element topology information define the co-simulation interface and a mapping between dissimilar surface meshes is performed. In this region the displacements are exported into the global coordinate systems from the FEA solver, and the pressures normal to the element surfaces are imported back (ABAQUS Simulia, 2013).



Figure 8: Numerical FSI co-simulation representation of wind tunnel experiments.

The C-beam was laminated with 4 plies as quasi-isotropic in the three surfaces $[\phi=0 / 45 /-45 / 0]$ and two bend-twist coupling plies on the top and bottom surfaces $[\phi=30 / 30]$. So the top surface laminate was modelled as $[\phi=0 / 45 /-45 / 0 / 30 / 30]$ from bottom to top. The finite element model of the foil section closely represents the wind tunnel specimen, Figure 9(a). The material properties of the carbon, foam and Mylar were tested independently at the University of Southampton (Marimon Giovannetti, 2017).

A summary of the material properties used in the FEA analysis is found in Table 1. 
Table 1: Material properties used in the FEA model.

\begin{tabular}{lllllll}
\hline Material & $\rho$ & $E_{11}$ & $E_{22}$ & $\nu$ & $\begin{array}{l}G_{12} \\
{[\mathrm{MPa}]}\end{array}$ & $\begin{array}{l}G_{13} \\
{[\mathrm{MPa}]}\end{array}$ \\
\hline \hline Carbon & 3 & 117940 & 7840 & 0.25 & 4400 & 3600 \\
Glass & & 38000 & 4100 & 0.35 & 4350 & 4350 \\
Foam & 0.0036 & 10.4 & 10.4 & 0.3 & 10.14 & 10.14 \\
Mylar & 2 & 2042.15 & & 0.38 & \\
\hline \hline
\end{tabular}

The CFD environment was also set to replicate the wind tunnel experiments, representing the same dimensions of the working section of the wind tunnel in the $y$ and $z$ directions (i.e. $3.5 \mathrm{~m} \mathrm{x} 2.4 \mathrm{~m}$ width and height of the R. J. Mitchell wind tunnel), see Figure 8. For the CFD domain it was also important to represent correctly the boundary layer mesh, shown in Figure 9 (b), and the transition along the span from the symmetric aerofoil section to the asymmetric one. As in the wind tunnel the flow was not planned to be tripped to be fully turbulent near the leading edge of the aerofoil, to avoid surface defects, the $\gamma R e_{\theta}$ transition model was used in the numerical CFD environment to closely represent the wind tunnel conditions. The GammaRe-Theta transition model is coupled to the $k$ - $\omega$ SST model through the use of the effective intermittency that in turn determines the percentage of time in which the flow is turbulent (Sorensen, 2009). The Gamma-Re-Theta model is generally used to accurately capture lift and drag on aerofoils, replicating the complex transition from laminar to turbulent flows happening near the leading edge of the foils. 
(a) FEA mesh and top surface lamination. FEA mesh size: 50,000 elements.

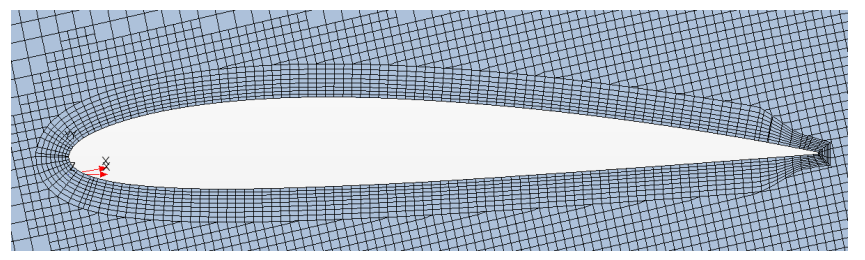

(b) CFD tip section boundary layer mesh. CFD number of cells: 4.8 million. Boundary layer thickness: $0.018 \mathrm{~m}$.

Figure 9: FSI numerical model mesh.

The settings used for the fluid-structure interaction numerical model are summarised in Table 2. 
Table 2: FSI numerical settings within Star-CCM+.

The aerofoil was manufactured, built and tested at the University of Southampton. Two internal spars were produced to understand the influence of passive adaptivity in different materials. One C-beam was made only of pre-preg unidirectional carbon plies (Gurit, 2012): in top and bottom faces $\phi=[0 / 45 /-45 / 0 / 30 / 30]_{C}$. The second beam was manufactured with pre-preg unidirectional quasi-isotropic E-Glass plies (PRF Composite Materials, 2015) and carbon PAC plies: in top and bottom faces $\phi=[0 / 45 /-45 / 0]_{G}+[30 / 30]_{C}$. During the manufacturing of the beams, the composite plies were layered, ensuring that the angle conventions were the same as the ones modelled 
numerically.

The structure was tested in the R. J. Mitchell closed circuit wind tunnel. The internal spar was fixed to the dynamometer via a Rexroth ${ }^{\circledR}$ frame, as described in (Banks et al., 2015; Marimon Giovannetti et al., 2017).

For the tests on the foil, both DIC and PIV were high-speed systems. All four cameras and the laser were positioned in the viewing room, as can be seen in Figure 10. The same DIC set up used in (Marimon Giovannetti et al., 2017) was used in the new experimental set-up. The high-speed stereo PIV equipment is detailed in Table 3.

Table 3: PIV performance table showing the equipment and the setting used.

\begin{tabular}{ll}
\hline Equipment & Set-up \\
\hline \hline & 2 Phantom v xx1 \\
& Sensor size: $25.6 \times 16 \mathrm{~mm}$ \\
& Pixel size: $10 \mu \mathrm{m}$ \\
& Resolution $(\max ): 2560 \times 1600$ pixels \\
& Exposure time: $2498 \mu \mathrm{s}$ \\
Camera & Frame rate: $0.2-0.4 \mathrm{kHz}$ \\
& Stereo angle: $\approx 39.5 \mathrm{deg}$. \\
& $\Delta t=60-40-30-25-20 \mu \mathrm{s}$ for $V=10-15-20-25-30 \mathrm{~m} / \mathrm{s}$ \\
\hline & Nikon $200 \mathrm{~mm} \mathrm{f} 4$ \\
& Aperture: $f-4$ \\
& Depth of field: $16 \mathrm{~mm}$ \\
& LD75-G PIV High repetition rate DPSS Nd:YAG \\
& Wavelength: $532 \mathrm{~nm}$ \\
Laser & Output energy: $7.5 \mathrm{~mJ}$ at $10 \mathrm{kHz}$ \\
&
\end{tabular}

Figure 10(a) shows a schematic drawing of the equipment in the wind tunnel. The high-speed laser was positioned in the viewing room and the laser beam was deviated through two mirrors angled at $45^{\circ}$ to obtain a focused sheet positioned $1 / 3$ of chord downstream of the trailing edge. The PIV calibration was performed calibrating the left-had camera (Cam1) with the front of the calibration plate and the right-hand camera (Cam2) with the back of the calibration plate. 


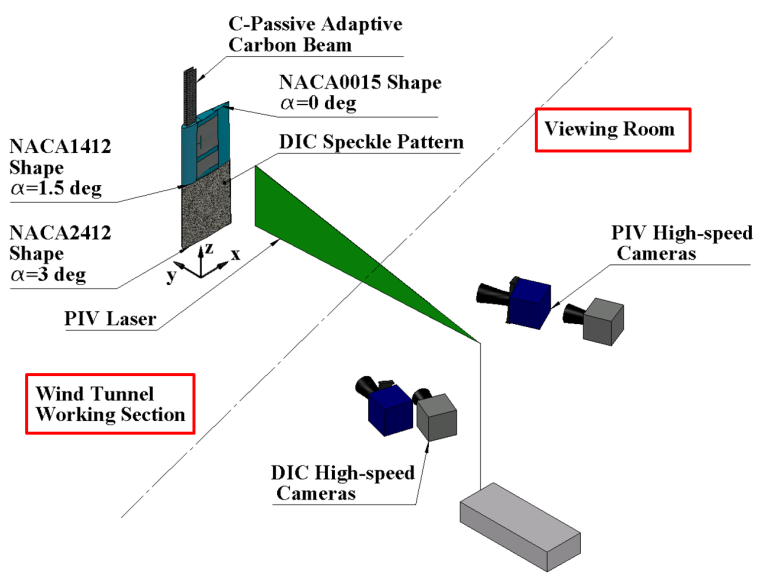

(a) Wind tunnel set up drawing

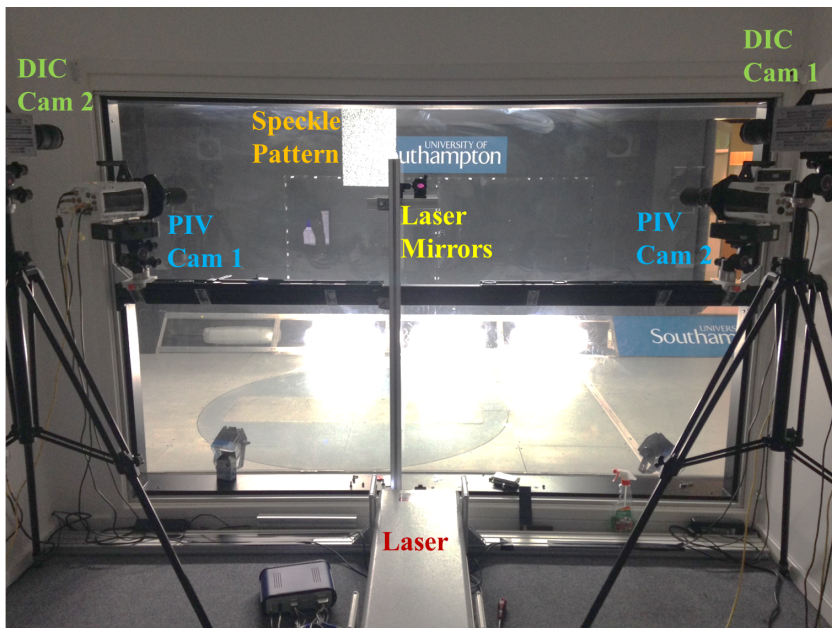

(b) Viewing room set-up

Figure 10: Wind tunnel and equipment set-up.

Having set the laser in the viewing room together with the DIC cameras, in order to ensure optical isolation between the two systems, it was necessary to apply a filter on the DIC cameras with transmittance of $0 \%$ in the greenwavelength region. The R-60 filter by (Edmund Optics, 2016) was installed 
on the DIC cameras. The LED lights were also covered with magenta gel filters to allow the high-speed DIC cameras to only detect two-thirds of the white light histogram, cutting off the laser wavelength. Moreover, two lowpass $532 \mathrm{~nm} \pm 10 \mathrm{~nm}$ filters were applied to the PIV high-speed cameras to allow them to only capture the laser wavelength. The high speed setup of both DIC and PIV systems allows to capture the structure response to fluid loading and the flow features around the aerofoil simultaneously in a dynamic environment such as the wind tunnel. This allows for further understanding of complex interactions occurring between fluids and structures.

\section{FSI results}

Figure 11 presents the lift force response for $\alpha=10.02^{\circ}$ and a range of wind speeds. The two internal structures are compared. It is possible to see that the glass-carbon C-beam, being more flexible and prone to twist, allows a larger reduction in lift over velocity slope. This response is possible due to the larger bend-twist coupling effects occurring in the structure with less bending and torsional stiffness.

Both the numerical and wind tunnel data show that it is possible to design a structure tailored to a design goal controlling the level of bend-twist coupling. The presented results significantly reduce the increase in lift force with speed, ensuring that is possible to use numerical FSI methods to aid the design of tailored structures. Designing for the inherent flexibility of a foil structure, it is possible to change the lift to velocity ratio, which for rigid quasi-isotropic aerofoil would change as a function of $V^{2}$. The influence of both the deflection and the twist affect the aerodynamic response and viceversa. For this reason it is important to understand and accurately measure the interactions between the flow features and the structure.

The lower torsional rigidity of the glass fibres leads to an increase in twist values (Figure 12). From both figures it is possible to see how the FSI numerical model correctly captures the twist and the aerodynamic response of the aerofoil with a carbon spar. The FSI numerical model presents a slightly higher torsional stiffness (maximum twist error at $V=25 \mathrm{~m} / \mathrm{s}$ of $7 \%$ - absolute error of $0.1^{\circ}$ ) due to the discrepancies in the internal spar dimensions and the fillet of the C-spar edges manufactured to easily slide into the leading edge of the foil.

For high performance sailing boats such as the International Moth, it is necessary to achieve large changes in angle of attack at high boat speed 
even at the lowest set-angles of attack. For a set angle of attack of $5.1^{\circ}$ the maximum change in angle of attack is $1.695^{\circ}$, leading to a reduction in angle of attack of $\approx 33 \%$.



Figure 11: Lift force over wind speed for $\alpha=10.02^{\circ}$ : analytical, numerical FSI and wind tunnel results. 



Figure 12: Change in angle of attack for different wind speeds and set-angles of attack at $90 \%$ of aerofoil span. The solid lines represent the carbon spar and the dotted lines represent the glass-carbon spar as measured in the wind tunnel. For $\alpha=20.04^{\circ}$ the closed marker indicates the carbon beam and the open marker the glass-carbon beam. The green markers presented for $\alpha=10.02^{\circ}$ show the FSI twist for the modelled aerofoil with a carbon spar.

The foil can passively adjust its angle of attack to reduce the induced drag associated with the foiling configuration and the lift once reached the take-off speed. Figure 13(a) shows that for the more flexible glass internal spar, for $V=15 \mathrm{~m} / \mathrm{s}$ and $\alpha=14.95^{\circ}$, the aerofoil twist is increased by $\approx 13 \%$ and therefore a reduction in drag of $\approx 13 \%$ is seen. In Figure 13 (a) the wind tunnel and CFD results are also presented. It is possible to see that for the whole range of angles of attack the aerofoil with the internal Carbon-Glass spar has a lower drag coefficient due to the change in angle of attack that results in a decrease in induced drag. Moreover, the aerofoil with the internal Carbon-Glass spar presents a higher $C_{L} / C_{D}$ meaning that the reduction in induced drag has more impact than the reduction in lift due to deflection. 




(a) Drag coefficient over angle of attack.



(b) $C_{L} / C_{D}$ over angle of attack.

Figure 13: Numerical CFD and wind tunnel results for $V=15 \mathrm{~m} / \mathrm{s}$. 
A comparison of a PAC model with a quasi-isotropic structure of a similar aerofoil was presented in (Marimon Giovannetti et al., 2016). From Figure 14 it is possible to see that the ratio of tip deflection between a quasi-isotropic and a PAC for a range of angles of attack and wind speeds varies non-linearly. The PAC structure, with its fibres oriented off-axis, is more prone to bend, with an increase of tip deflection ranging between $5 \%$ and $30 \%$. The nonlinear behaviour of bend-twist coupling structure requires the use of accurate measures developed using full-field imaging techniques to correctly capture the deflection of the whole tip of the foil and its influence on the flow around it.

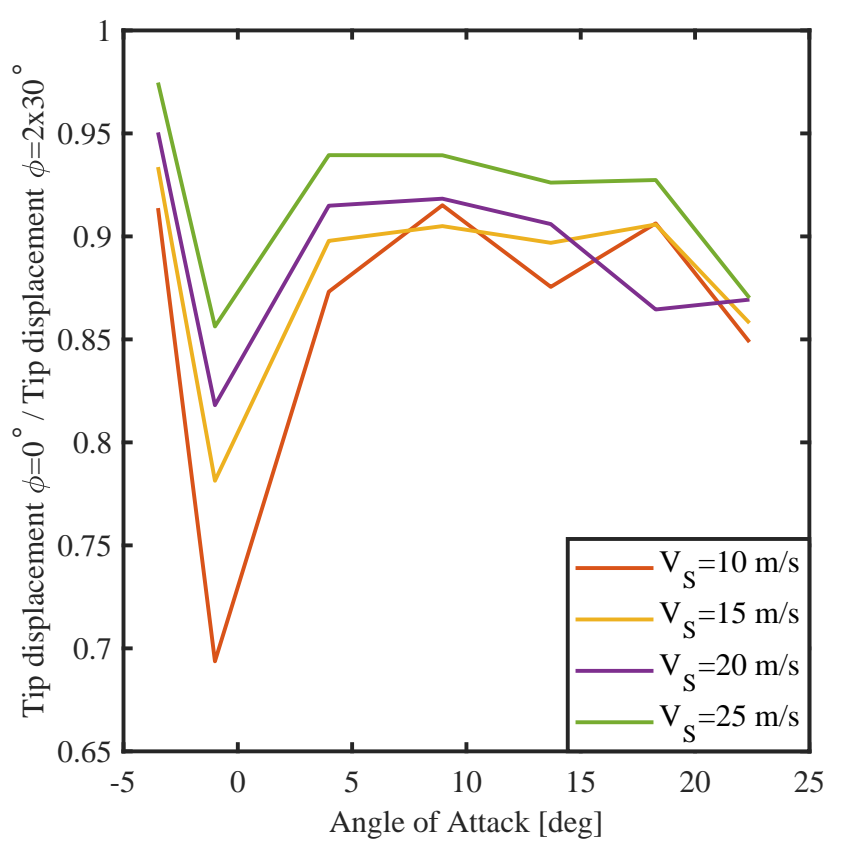

Figure 14: Tip deflection ratio between a quasi-isotropic and a bend-twist coupling structure for a range of angles of attack and wind speeds.

\section{Conclusions}

A fluid-structure interaction numerical model was developed to allow the design and evaluation of passively adaptive hydrofoils for high-performance sailing boats. This combined an FEA model of a bend-twist coupled composite foil structure with a RANS CFD model to predict the performance 
of flexible foils. The numerical method was used to design a simplified hydrofoil geometry that passively reduces its angle of attack as the flow speed increased. To validate this method the designed foil structure was tested in a wind tunnel with full field deformation measurements. A close agreement was found between the numerical and experimental results validating the numerical methodology. The simplified test case has demonstrated that is possible to design a structure tailored to a certain load. The increase in lift with velocity is significantly reduced compared to a rigid section. The glass/carbon-fibre C-beam, with a lower torsional and bending stiffness compared to the full-carbon one, can achieve changes in angles of attack of almost 4 degrees for $V=25 \mathrm{~m} / \mathrm{s}$ and $\alpha=14.95^{\circ}$. This leads to a reduction of $\approx 13 \%$ on the drag and an increase in $C_{L} / C_{D}$ ratio. The results from the numerical simulations and experimental tests show that the developed tools are able to capture the complex interactions between a fluid and a structure and the importance of capturing the deflections and twists of foils when analysing their performances. The next step required is to investigate and validate the dynamic response of passive adaptive foils to allow such techniques to be used to improve the performance of sailing foils in the future.

\section{Acknowledements}

The authors would like to acknowledge the EPSRC for funding this research under the grant number EP/I009876/1. The authors would also thank the University of Southampton, the members of the TSRL and Dave Marshall and his team in the R. J. Mitchell wind tunnel. Moreover, we would like to thank Dr Nila from LaVision for his assistance with the DIC and PIV set-up.

\section{References}

ABAQUS Simulia (2013). ABAQUS Analysis User's Guide. Technical report.

Banks, J., L. Marimon Giovannetti, X. Soubeyran, A. Wright, S. R. Turnock, \& S. W. Boyd (2015). Assessment of Digital Image Correlation as a method of obtaining deformations of a structure under fluid load. Journal of Fluids and Structures 58, 173-187.

Banks, J., L. Marimon Giovannetti, J. Taylor, \& S. R. Turnock (2016). Assessing Human-Fluid-Structure Interaction for the International Moth. Procedia Engineering 147, 311-316. 
Barber, R. B. (2017). Adaptive Pitch Composite Blades for Axial-Flow Marine Hydrokinetic Turbines. Phd thesis, University of Washington.

Beaver, B. \& J. Zseleczky (2009). Full scale measurement on a hydrofoil International Moth. In 19th Cheasap. Sail. YACHT Symp.

Chen, F., X. Chen, X. Xie, X. Feng, \& L. Yang (2013, September). Fullfield 3D measurement using multi-camera digital image correlation system. Optics and Lasers in Engineering 51 (9), 1044-1052.

Crammond, G., S. Boyd, \& J. Dulieu-Barton (2013, December). Speckle pattern quality assessment for digital image correlation. Optics and Lasers in Engineering 51(12), 1368-1378.

de Borst, R., P. Nithiarasu, T. Tezduyar, G. Yagawa, \& T. Zohdi (2013). Computational Fluid-Structure Interaction. John Wiley \& Sons, Ltd., Publication.

De Goeij, W. C., M. J. L. Van Tooren, \& A. Beukers (1999). Implementation of bending-torsion coupling in the design of a wind-turbine rotor-blade. Applied Energy 63(3), 191-207.

Donea, J., S. Guiliani, \& J. P. Halleux (1982). An Arbitrary-LagrangianEulerian finite element method for transient dynamic fluid-structure interaction. Computer Methods in Applied Mechanics and Engineering 33, 689-723.

Ducoin, A., J. André Astolfi, \& J. F. Sigrist (2012). An experimental analysis of fluid structure interaction on a flexible hydrofoil in various flow regimes including cavitating flow. European Journal of Mechanics, B/Fluids 36, 63-74.

Edmund Optics (2016).

Fedorov, V. (2012). Bend-Twist Coupling Effects in Wind Turbine Blades. Ph. D. thesis, Technical University of Denmark.

Grediac, M. \& F. Hild (2012). Full-Field Measurements and Identification is Solid Mechnics. John Wiley \& Sons.

Gurit (2012). SE 84 LV Low temperature cure epoxy prepreg. Technical report. 
Herath, M. T., A. K. L. Lee, \& B. Gangadhara Prusty (2015). Design of shape-adaptive wind turbine blades using Differential Stiffness Bend-Twist coupling. Ocean Engineering 95, 157-165.

Hou, G., J. Wang, \& A. Layton (2012). Numerical Methods for FluidStructure Interaction - A Review. Communications in Computational Physics 12(2), 337-377.

Hu, H., M. Tamai, \& J. T. Murphy (2008, September). Flexible-Membrane Airfoils at Low Reynolds Numbers. Journal of Aircraft 45(5), 1767-1778.

International Moth Class (2013). International Moth Class Rules. Technical report.

Jones, G. S., J. C. Lin, B. G. Allan, W. E. Milholen, C. L. Rumsey, \& R. C. Swanson (2008). Overview of CFD Validation Experiments for Circulation Control Applications at NASA. In International Powered Lift Conference, pp. 1-16.

Khan, A., D. Adams, V. Dayal, \& J. Vogel (2000). Effects of bend-twist coupling on composite propeller performance. Mechanics of Composite Materials and Structures 7, 383-401.

Lin, H.-J. \& W.-M. Lai (2010, May). A Study of Elastic Coupling to the Wind Turbine Blade by Combined Analytical and Finite Element Beam Model. Journal of Composite Materials 44 (23), 2643-2665.

Maheri, A. \& A. T. Isikveren (2009). Design of wind turbine passive smart blades. In European Wind Energy Conference.

Malijaarsl, P. J. \& M. L. Kaminski (2015). Hydro-elastic Analysis of Flexible Propellers: an overview. In Fourth International Symposium on Marine Propulsors, Austin.

Marimon Giovannetti, L. (2017). Fluid structure interaction testing, modelling and development of Passive Adaptive Composite foils. Phd thesis, University of Southampton.

Marimon Giovannetti, L., J. Banks, S. R. Turnock, \& S. W. Boyd (2016). Developing tools for assessing the Fluid Structure Interaction of Passive Adaptive Composite foils. In 6th International Conference on Structural Engineering Mechanics and Computation. 
Marimon Giovannetti, L., J. Banks, S. R. Turnock, \& S. W. Boyd (2017). Uncertainty assessment of coupled Digital Image Correlation and Particle Image Velocimetry for fluid-structure interaction wind tunnel experiments. Journal of Fluids and Structures 68, 125-140.

Mccormick, N. (2012). Digital image correlation for structural measurements. Proceedings of the Institution of Civil Engineers 165, 185-190.

Nicholls-Lee, R., S. W. Boyd, \& S. R. Turnock (2009). Development of high performance composite bend-twist coupled blades for a horizontal axis tidal turbine. In 17th International Conference on Composite Materials.

PRF Composite Materials (2015). Toughened Epoxy Prepreg System RP528: low-medium temperature cure 85-120 deg C. Technical report.

Raffel, M., C. Willert, \& S. Wereley (2007). Particle Image Velocimetry - A Practical Guide.

Raither, W., A. Bergamini, \& P. Ermanni (2012). Profile beams with adaptive bending-twist coupling by adjustable shear centre location. Journal of Intelligent Material Systems and Structures 24, 334-346.

Rastogi, P. \& E. Hack (2012). Optical Methods for Solid Mechanics: A FullField Approach.

Siddiqui, M. Z. (2014). 2D-DIC for the quantitative validation of FE simulations and non-destructive inspection of aft end debonds in solid propellant grains. Aerospace Science and Technology 39, 128-136.

Sigrist, J.-F. (2015). Fluid-Structure Interaction an introduction to finite element coupling. Wiley.

Sorensen, N. N. (2009). CFD Modelling of Laminar-turbulent Transition for Airfoils and Rotors Using the $\gamma-R e_{\theta}$ Model. Wind Energy 12, 715-733.

Star-ccm + (2015). Star-ccm+ user guide. Technical report.

Tamai, M., J. T. Murphy, \& H. Hu (2008). An Experimental Study of Flexible Membrane Airfoils at Low Reynolds Numbers. 46th AIAA Aerospace Sciences Meeting and Exhibit, 1-12. 
Tang, Z., J. Liang, Z. Xiao, \& C. Guo (2012, February). Large deformation measurement scheme for 3D digital image correlation method. Optics and Lasers in Engineering 50(2), 122-130.

Veers, P., S. N. Laboratories, G. Bir, N. Renewable, N. Wind, \& D. Lobitz (1998). Aeroelastic Tailoring in Wind-Turbine Blade Applications. Wind Energy.

Wieneke, B. (2014). Generic a-posteriori uncertainty quantification for PIV vector fields by correlation statistics. In 17th International Symposium on Applications of Laser Techniques to Fluid Mechanics, pp. 7-10.

Young, Y. L. \& M. R. Motley (2011). Influence of Material and Loading Uncertainties on the Hydroelastic Performance of Advanced Material Propellers. In Second International Symposium on Marine Propulsors. 
Click here to download high resolution image

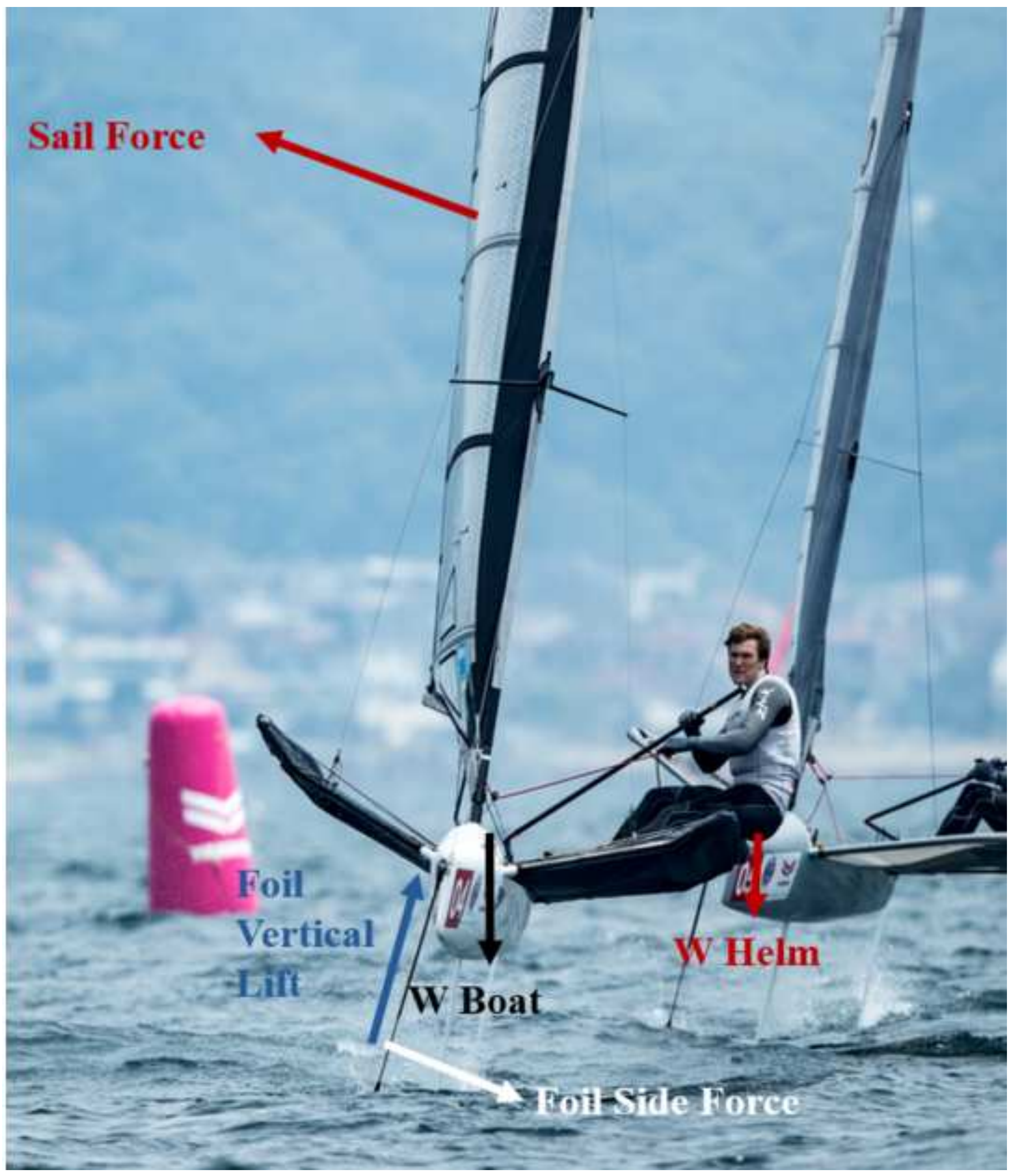




\section{PAC fixed foil}

\section{Moving elevator}

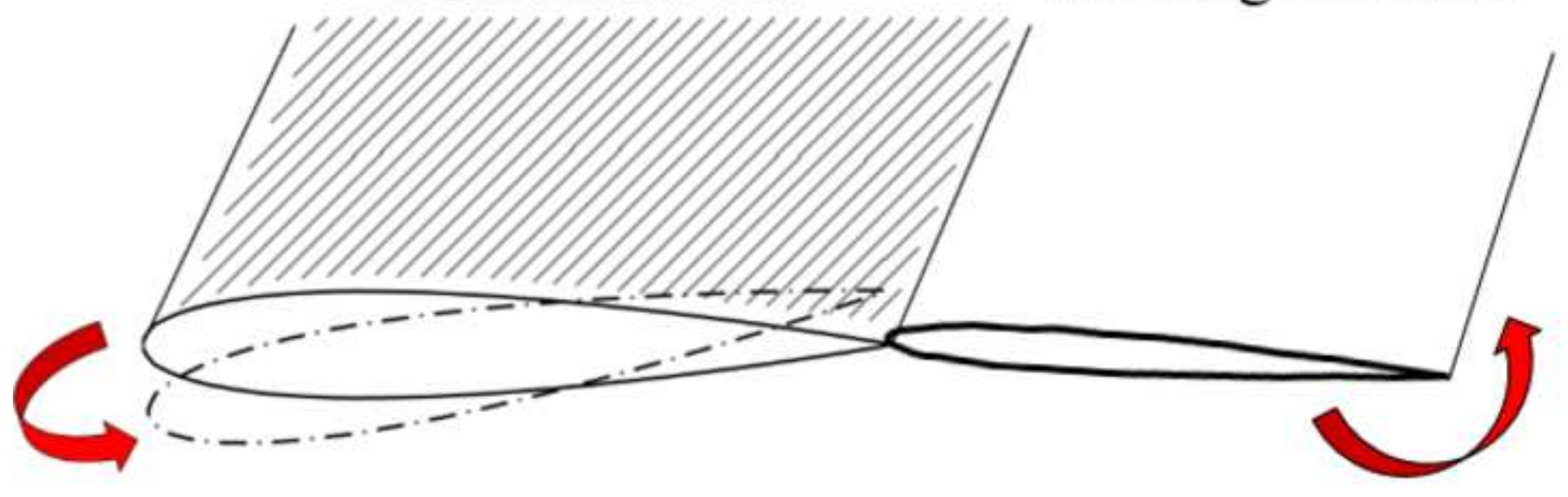











Figure4 




Angle of Attack 
Figure 7
Click here to download high resolution image

\section{Side View}



\section{Top View}

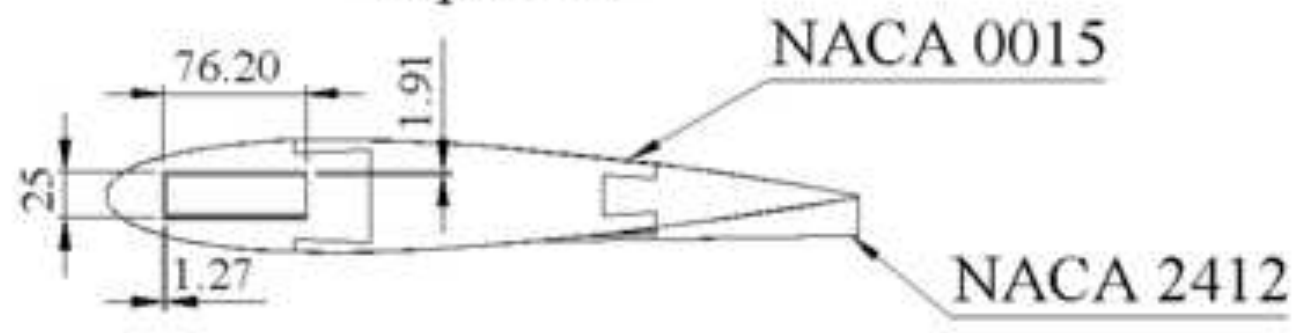

NACA 0015

Bottom View

NACA 2412 
Click here to download high resolution image

\section{STAR-CCIM-}




Click here to download high resolution image



\section{C-Beam Top Section} Lamingtion






\section{igure $9 b$}

Click here to download high resolution image






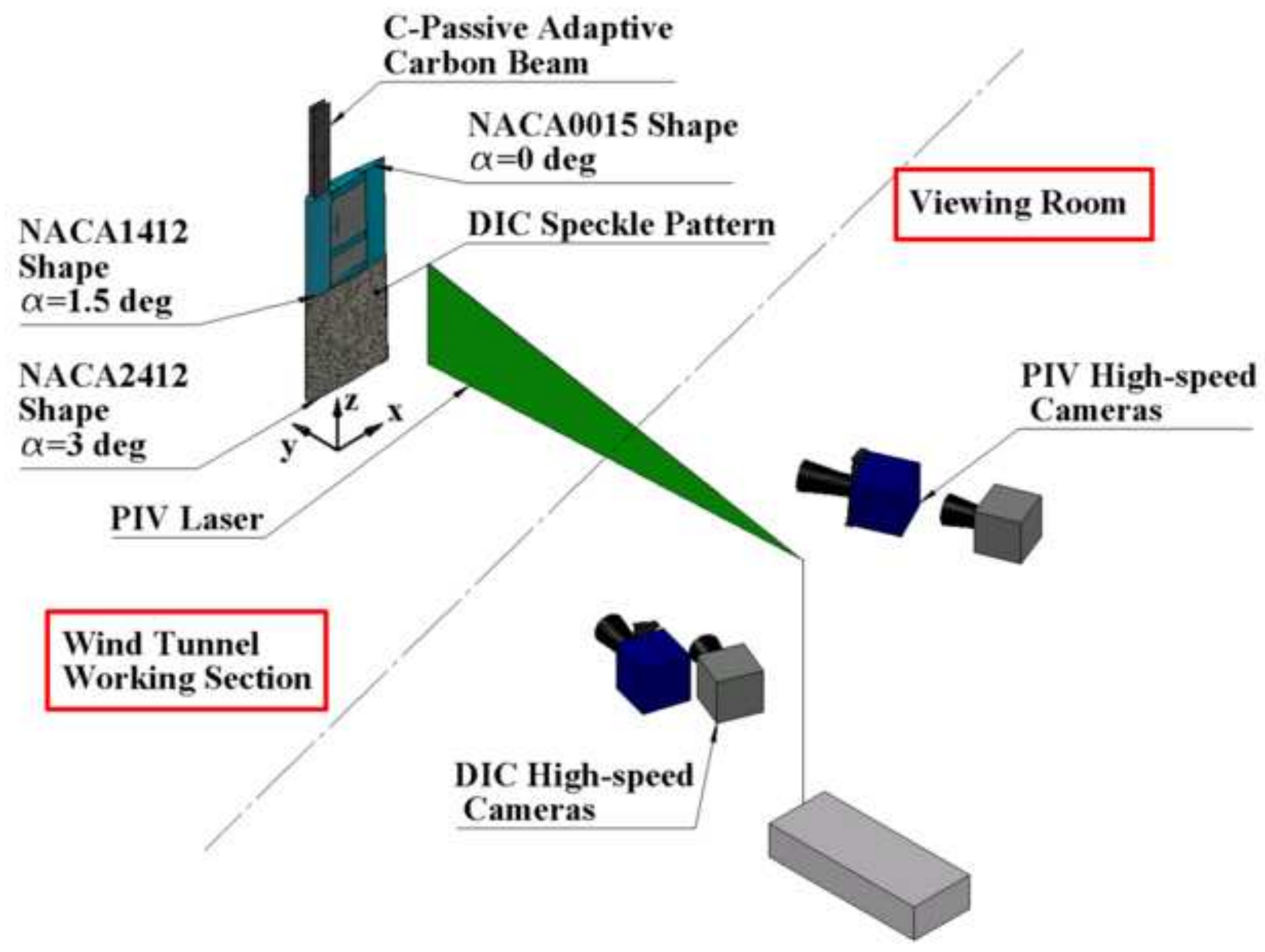


Click here to download high resolution image






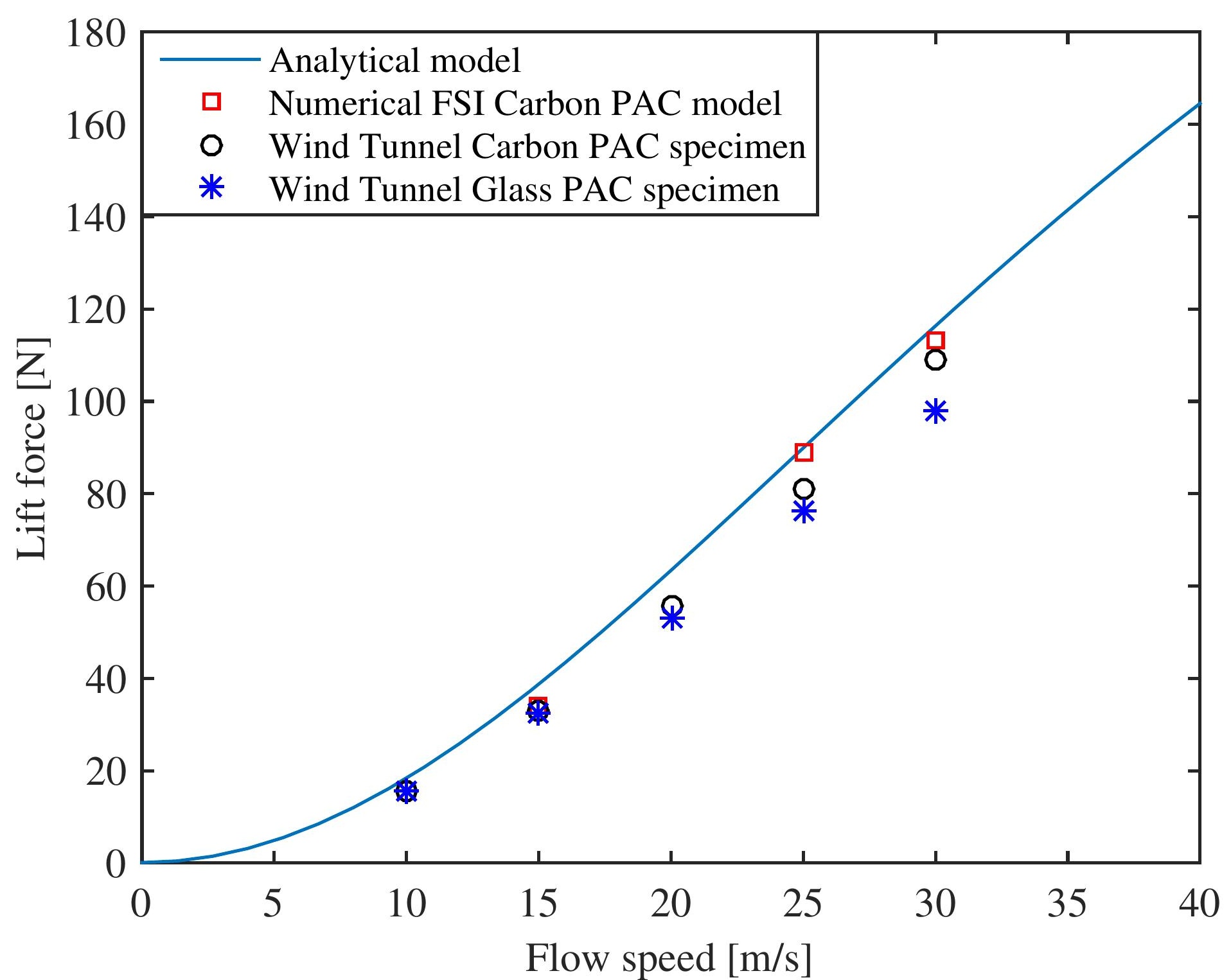


Figure12
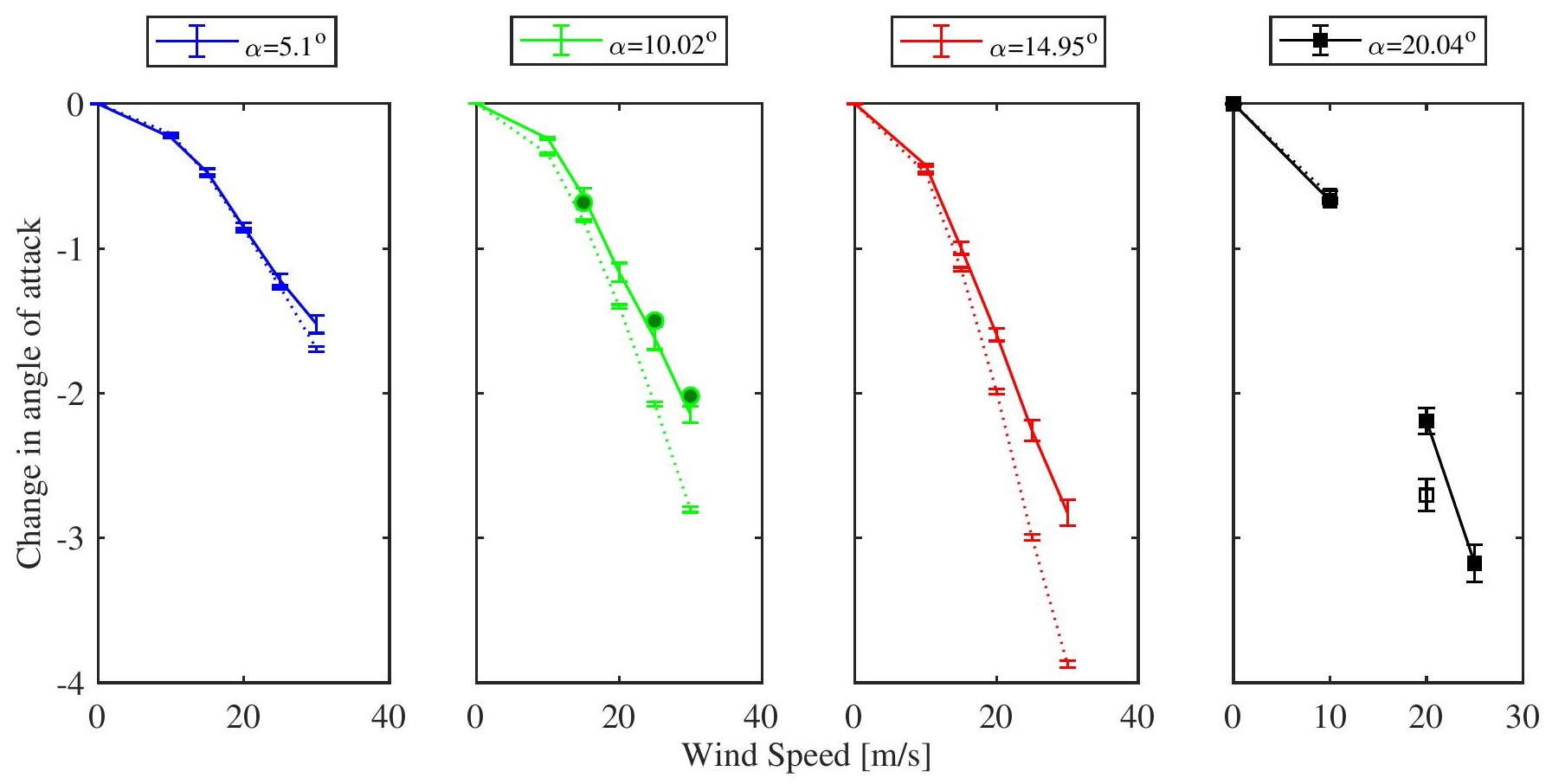


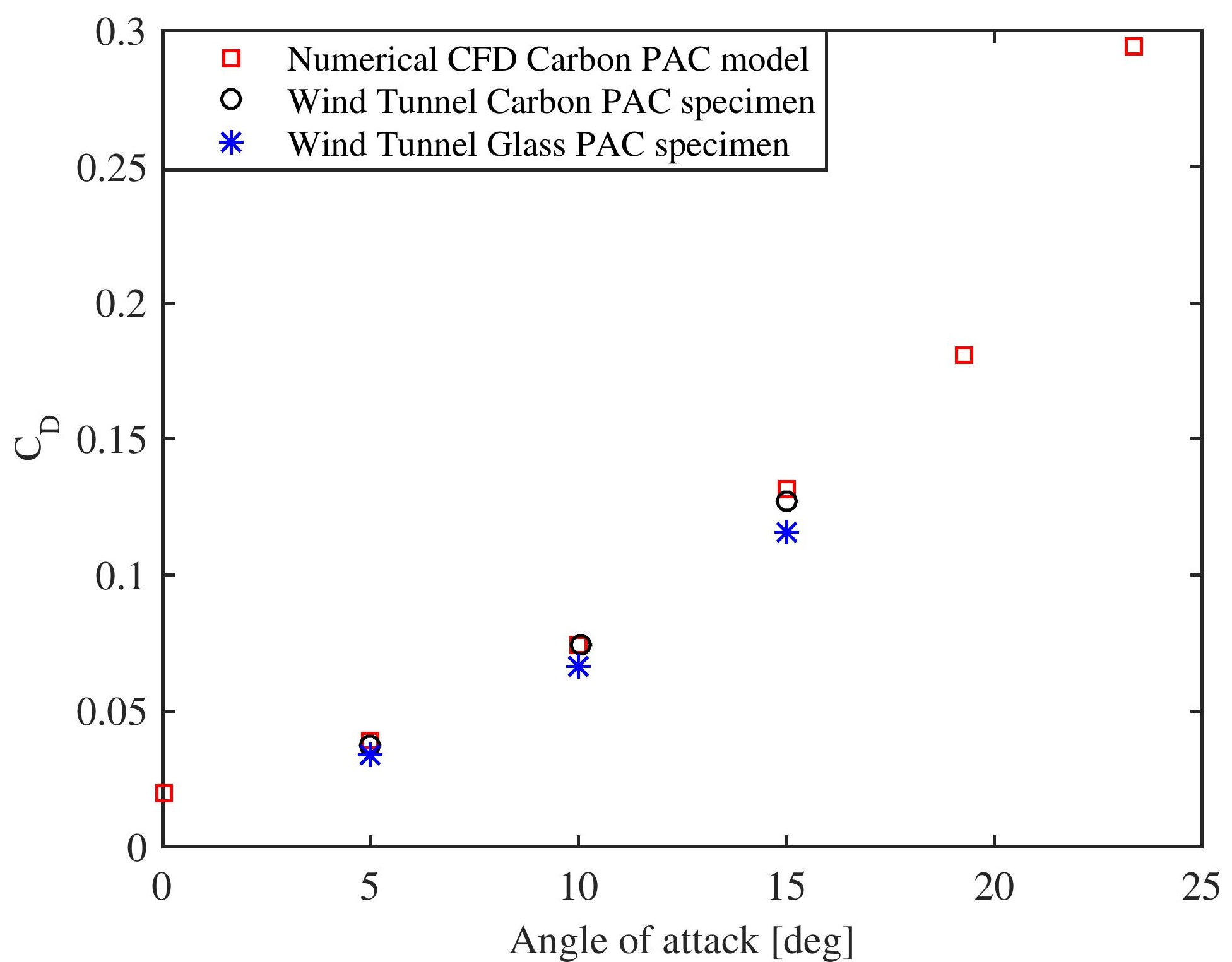









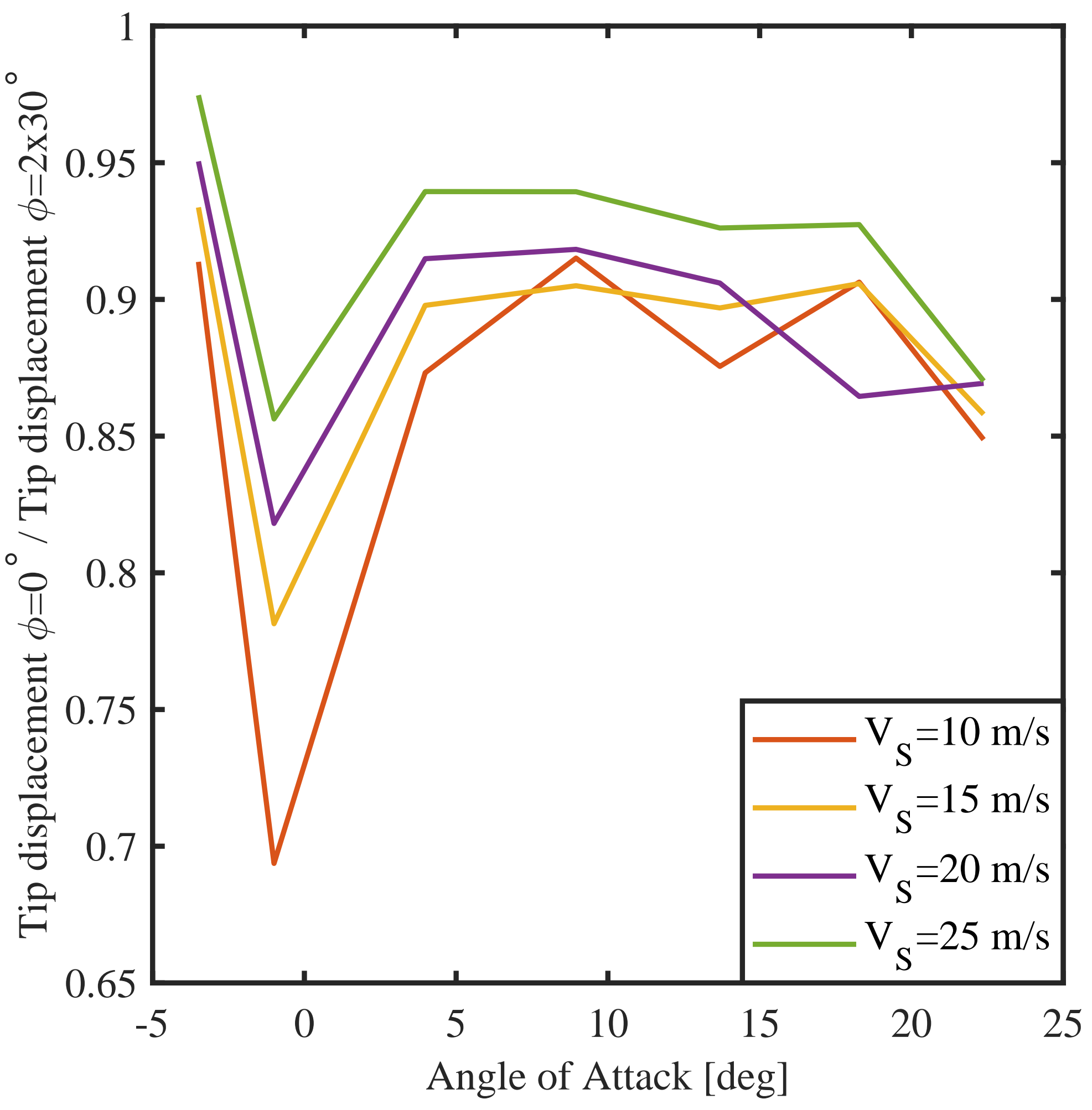


Click here to download LaTeX Source Files: journal_OceanEngineering_v4.tex 
bib
Click here to download LaTeX Source Files: biblnnovsail.bib Click here to download LaTeX Source Files: biblnnovsail bib

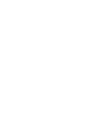


(1) (1) (1) (1) (1) (1) (1) (1)



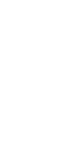


. . .

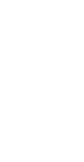



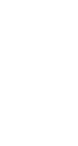

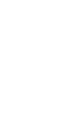





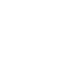





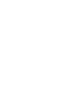

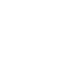


.

(1) 
elsarticle
Click here to download LaTeX Source Files: elsarticle.cls





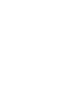

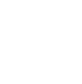


.


. . .





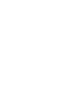


更 . . . 更 更 更 更 更 更 更 
Click here to download LaTeX Source Files: chicaco.sty Click here to download LaTeX Source Files: chicaco.sty

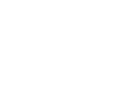

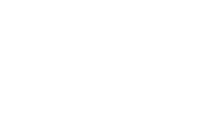

(1)
(1)

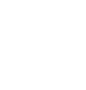
(1) (1) (1)

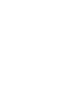
(1)



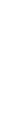
更

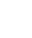
.





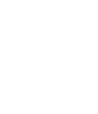



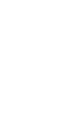





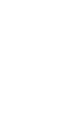







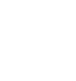
(1)

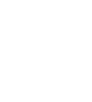



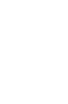

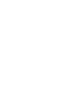





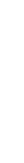


.



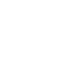

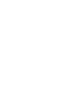





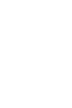







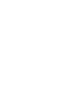



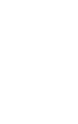







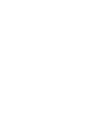

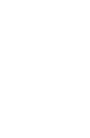
. . 